\title{
Differential Connectivity Within the Parahippocampal Place Area
}

\author{
Christopher Baldassano ${ }^{\mathrm{a},{ }^{*}, \text { Diane M. Beck }}{ }^{\mathrm{b}}$, and Li Fei-Fei ${ }^{\mathrm{a}}$ \\ aDepartment of Computer Science, Stanford University, Stanford, CA, USA \\ ${ }^{b}$ Beckman Institute and Department of Psychology, University of Illinois at Urbana-Champaign, \\ Urbana, IL, USA
}

\begin{abstract}
The Parahippocampal Place Area (PPA) has traditionally been considered a homogeneous region of interest, but recent evidence from both human studies and animal models has suggested that PPA may be composed of functionally distinct subunits. To investigate this hypothesis, we utilize a functional connectivity measure for fMRI that can estimate connectivity differences at the voxel level. Applying this method to whole-brain data from two experiments, we provide the first direct evidence that anterior and posterior PPA exhibit distinct connectivity patterns, with anterior PPA more strongly connected to regions in the default mode network (including the parieto-medial temporal pathway) and posterior PPA more strongly connected to occipital visual regions. We show that object sensitivity in PPA also has an anterior-posterior gradient, with stronger responses to abstract objects in posterior PPA. These findings cast doubt on the traditional view of PPA as a single coherent region, and suggest that PPA is composed of one subregion specialized for the processing of low-level visual features and object shape, and a separate subregion more involved in memory and scene context.
\end{abstract}

\section{Keywords}

Parahippocampal Place Area; PPA; Functional Connectivity; fMRI

\section{Introduction}

Over the past two decades, functional magnetic resonance imaging (fMRI) has identified a number of category-selective regions involved in visual processing. Most of these regions have been defined based on differential activation to one category of stimuli over another, but this hypothesis-driven approach to mapping brain regions has significant drawbacks. Adjacent areas that have similar response profiles to the presented stimuli, but different functions, may be mistakenly conflated; for example, functionally distinct subregions have been identified in both object-sensitive lateral occipital complex (LOC) (Grill-Spector et al. 1999) and the extrastriate body area (Weiner and Grill-Spector 2011).

Another visual region that has been proposed as a candidate for subdivision is the Parahippocampal Place Area (PPA) (Epstein and Kanwisher 1998). This scene-sensitive

\footnotetext{
(C) 2013 Elsevier Inc. All rights reserved.

*Corresponding author. Department of Computer Science, Stanford University, 353 Serra Mall, Stanford, CA 94305. chrisb33@cs.stanford.edu (C. Baldassano). Fax: +1 6507257411.
}

Publisher's Disclaimer: This is a PDF file of an unedited manuscript that has been accepted for publication. As a service to our customers we are providing this early version of the manuscript. The manuscript will undergo copyediting, typesetting, and review of the resulting proof before it is published in its final citable form. Please note that during the production process errors may be discovered which could affect the content, and all legal disclaimers that apply to the journal pertain. 
area has been heavily implicated in visual scene perception, though the precise nature of the representation in this area has been controversial. Leading models have argued that PPA represents local scene geometry (Epstein et al. 2003), spatial expanse (Park et al. 2011, Kravitz et al. 2011a), space-defining objects (Mullally and Maguire 2011), or contextual relationships (Bar 2004). All of these models have implicitly assumed that PPA is a homogeneous unit performing a single functional role, but this view has recently been called into question. In the last several years, a number of researchers have suggested that PPA could have multiple functional components. Differences in spatial frequency response (Rajimehr et al. 2011), varying deficits resulting from PPA lesions (Epstein 2008), PPA's overlap with multiple visual field maps (Arcaro et al. 2009), and a clustering meta-analysis (Sewards 2011) all hint at the possibility that PPA may be comprised of at least two functionally distinct subunits along its posterior-anterior axis. However, studies explicitly searching for a distinction between posterior and anterior PPA have failed to identify major differences (Cant and Xu 2012; Epstein and Morgan 2012).

Anatomical data from a proposed macaque homologue of PPA presents an interesting possibility for identifying subregions of human PPA. Although the definition of macque PPA is still a matter of ongoing research (Rajimehr et al. 2011; Nasr et al. 2011; Sewards 2011), a possible candidate spans cytoarchitectonically defined parahippocampal areas TH, TF, and TFO (Kravitz et al. 2011b). The most anterior area, TH, is primarily connected to retrosplenial cortex (RSC) (Suzuki 2009; Kravitz et al. 2011b) and is also connected to the caudal inferior parietal lobule (cIPL) through a parieto-medial temporal pathway (Cavada and Goldman-Rakic 1989; Kravitz et al. 2011b). The more posterior TF is connected to a similar set of regions, but receives stronger input from ventral visual areas V4 and TEO (Suzuki 2009). The specific connectivity properties of the most posterior area (TFO) are not yet known, but it has been shown that TFO has a neuronal architecture highly similar to that of ventral visual regions (Saleem et al. 2007). In short, these macaque parahippocampal regions exhibit an anterior-posterior gradient, with the anterior side most related to RSC and cIPL and the posterior side most related to ventral visual areas.

Connectivity results in humans, using both diffusion tensor imaging (DTI) and fMRI, have shown that the parahippocampal region is connected to occipital visual cortex (Kim and Kim 2005; Rushworth et al. 2006; Libby et al. 2012) as well as RSC and posterior parietal cortex (Rushworth et al. 2006; Kahn et al. 2008; Caspers et al. 2011; Uddin et al. 2010; Libby et al. 2012), and PPA is known to combine both spatial and object identity information (Harel et al. 2012). However, it is not known whether the posterior and anterior parts of the PPA connect differentially to these two networks. If human PPA corresponds to some or all of the macaque areas TH/TF/TFO, it should be possible to identify an anterior-posterior gradient in the functional connectivity properties of PPA. Such a finding would not only reinforce the proposed link between PPA and these macaque parahippocampal regions, but also demonstrate that PPA is actually composed of at least two regions operating on different types of visual information, shedding new light on the controversy over its functional properties.

To test whether voxels within PPA have differing connectivity properties, we apply our recent method for learning voxel-level connectivity maps (Baldassano et al. 2012). Unlike standard functional connectivity measures that examine each voxel independently, our method considers all PPA voxels simultaneously to identify subtle differences in connectivity between voxels. After examining how several predefined ROIs connect to PPA, we perform a whole-brain searchlight analysis to identify the distinct cortical networks that connect preferentially to anterior or posterior PPA. We then demonstrate that these connectivity gradients are paired with gradients in functional selectivity, by evaluating the response to scenes and objects across PPA. Finally, we show that the connectivity gradients 
within PPA extend beyond PPA's borders, placing PPA in the context of ventral occipital and parahippocampal regions.

\section{Materials and Methods}

\subsection{Regularized Connectivity Method}

Investigating our hypothesis requires a method that characterizes functional connectivity patterns within a region of interest (ROI), at the voxel level. A number of studies have used fMRI functional connectivity measures to investigate structure within ROIs (Roy et al. 2009; Margulies et al. 2007; Kim et al. 2010; Cohen et al. 2008; Zhang et al. 2008; Chai et al. 2009; Margulies et al. 2009), but most previous approaches either do not measure connectivity at the voxel level (requiring spatial downsampling to a small number of subregions) and/or learn connectivity weights separately for each voxel (decreasing sensitivity and making comparisons between voxels more difficult). In our datasets, the PPA connectivity effects are too subtle to be detected by learning weights separately for each voxel (see Supplementary Fig. 1), and require the use of a method which can learn voxellevel connectivity maps that consider all voxels simultaneously. Support vector regression can learn these type of voxel-level connectivity maps (Heinzle et al. 2011), but does not utilize information about the spatial arrangement of the voxels and therefore requires a relatively large amount of data. To address this issue, we developed a method for examining connectivity differences within ROIs that is specifically tailored to small training sets typical in the fMRI setting. This method has been shown to recover voxel-level connectivity properties more accurately and efficiently than previous approaches (Baldassano et al. 2012).

The most common type of analysis for computing functional connectivity between two regions $A^{1}$ and $A^{2}$ measures how well the mean of all voxel timecourses in $A^{1}$ predicts the mean timecourse in $A^{2}$. We generalize this approach to identify voxel-level connectivity differences, by learning a weighted mean over the voxel timecourses in $A^{1}$ that best predicts the mean timecourse in $A^{2}$. The learned weights of the voxels in $A^{1}$ will then indicate the strength of the functional connection between each voxel and region $A^{2}$. Simply allowing each voxel weight to be learned independently leads to severe overfitting on typical fMRI datasets, but fMRI data naturally satisfies some regularity assumptions that can constrain our model. In particular, voxel connectivity properties are likely to be spatially correlated, with nearby voxels typically having more similar connectivity properties than spatially distant voxels. This reflects a common view of cortical organization, and is especially applicable to blood-oxygen-level dependent (BOLD) signals such as fMRI, since the hemodynamic response is spatially smooth. To incorporate this assumption, we add a spatial regularization term to our model, which encourages each voxel in $A^{1}$ to have a connectivity weight similar to its spatially adjacent neighbors.

The learned connectivity maps are therefore a compromise between two objectives. Our first goal is to match the weighted average of the $A^{1}$ timecourses to the mean $A^{2}$ timecourse, by adjusting the weights. Our second goal is to make the weights spatially smooth, to prevent overfitting and allow our weights to generalize to independent data runs. The relative importance of this second goal is controlled by a hyperparameter $\lambda$, allowing us to trade off between having all weights be learned independently $(\lambda=0)$ and having all weights be identical $(\lambda=\infty)$.

Mathematically, the connectivity weights are learned by minimizing the convex optimization objective 


$$
\underset{a, b}{\operatorname{minimize}}\left\|\left(\boldsymbol{a}^{T} \cdot \boldsymbol{A}^{\mathbf{1}}+b\right)-\operatorname{mean}_{v}\left(\boldsymbol{A}^{\mathbf{2}}\right)\right\|_{2}^{2}+\lambda\|\boldsymbol{D} \cdot \boldsymbol{a}\|_{2}^{2}
$$

where $\boldsymbol{a}$ is the connectivity weight map, $b$ is a constant offset, $\boldsymbol{A}^{\mathbf{1}}$ and $\boldsymbol{A}^{\mathbf{2}}$ are the (\# voxels $\times$

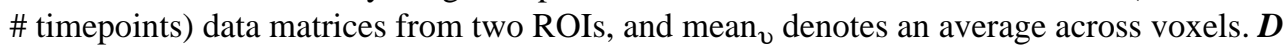
is the voxel connectivity matrix, which we design to penalize the mean squared difference between the weight $a_{i}$ of voxel $i$, and the weights of voxel i's neighbors:

$\|\boldsymbol{D} \cdot \boldsymbol{a}\|_{2}^{2}=\sum_{i=1}^{N} \frac{1}{\left|n_{i}\right|} \sum_{j \in n_{i}}\left(a_{i}-a_{j}\right)^{2}$ where $N$ is the number of voxels in $A^{1}$ and $n_{i}$ is the set of $i$ 's neighbors. The optimal a (for a given choice of $\lambda$ ) can be found efficiently by using a convex optimization package such as CVX (Grant and Boyd 2011). For further details and validation experiments, see Baldassano et al. (2012).

The following sections describe the collection of the datasets used to learn the connectivity weights $a$. As will be shown in the Results, PPA's functional connectivity properties are not sensitive to the choice of experimental dataset; the specific details of the stimuli and tasks in these experiments are provided only for reference purposes.

\subsection{Localizer and Object-in-Scene Experiments}

2.2.1. Participants -10 subjects ( 3 female) with normal or corrected-to-normal vision participated in the object-in-scene and localizer fMRI experiment. The study protocol was approved by the Stanford University Institutional Review Board, and all subjects gave their written informed consent.

2.2.2. Scanning Parameters-Imaging data were acquired with a 3 Tesla G.E. Healthcare scanner. A gradient echo, echo-planar sequence was used to obtain functional images [volume repetition time (TR), $2 \mathrm{~s}$; echo time (TE), $30 \mathrm{~ms}$; flip angle, $80^{\circ}$; matrix, $128 \times 128$ voxels; FOV, $20 \mathrm{~cm}$; 29 oblique $3 \mathrm{~mm}$ slices with $1 \mathrm{~mm}$ gap; in-plane resolution, $1.56 \times 1.56 \mathrm{~mm}]$. The functional data was motioncorrected and each voxel's mean value was scaled to equal 100 (no spatial smoothing was applied). We collected a high-resolution $\left(1 \times 1 \times 1 \mathrm{~mm}\right.$ voxels) structural scan (SPGR; TR, $5.9 \mathrm{~ms}$; TE, $2.0 \mathrm{~ms}$, flip angle, $\left.11^{\circ}\right)$ in each scanning session. The structural scan was used to calculate a transformation between each subject's brain and the Talairach atlas.

2.2.3. Localizer Stimuli and Procedure-For the localizer experiment, subjects performed 2 runs, each with 12 blocks drawn equally from six categories: child faces, adult faces, indoor scenes, outdoor scenes, objects (abstract sculptures with no semantic meaning), and scrambled objects (these stimuli have been used in previous studies such as Golarai et al. 2007). Images $(240 \times 240$ pixels; subtending $12.8 \times 12.8$ degrees of visual angle) were presented at fixation. Examples of scene and object stimuli are shown in Fig. 1a. Blocks were separated by $12 \mathrm{~s}$ fixation cross periods, and consisted of 12 image presentations, each of which consisted of a $900 \mathrm{~ms}$ image followed by a $100 \mathrm{~ms}$ fixation cross. Each image was presented exactly once, with the exception of two images during each block that were repeated twice in a row. Subjects were asked to maintain fixation at the center of the screen, and respond via button-press whenever an image was repeated. The total number of timepoints was 300 (150 per run).

2.2.4. Object-in-Scene Stimuli and Procedure-For the object-in-scene experiment, we presented two types of stimuli, as shown in Fig. 1b: (1) boats and cars on a blank white background (isolated objects); and (2) boats and cars with a street or water scene background (objects in context). Images $(450 \times 450$ pixels; subtending $24 \times 24$ degrees of 
visual angle) were presented 100 pixels (5 degrees) away from fixation in randomly determined directions. Subjects were informed that each image contained either a boat or a car, and were asked to indicate as quickly as possible whether the object was on the left half of the image or the right half of the image (using a button box). Subjects performed 4 runs, with 16 blocks per run (with a $14 \mathrm{~s}$ gap between blocks) and 9 images per block. The first 8 blocks of each run showed a boat or car placed in a photographic scene; for each block, the object could violate a semantic relationship (appearing in the wrong type of scene, e.g. a boat on a city street) and/or a geometric relationship (appearing in the wrong position in the scene, e.g. a car above a tree rather than on the street). Each presentation consisted of a 500 ms fixation cross, an image flashed for $100 \mathrm{~ms}$, a $300 \mathrm{~ms}$ mask, and then a $1300 \mathrm{~ms}$ response period (blank gray screen). The last 8 blocks of each run showed a boat or car on a white background; these images were identical to those presented in the first eight blocks, with the backgrounds removed (and presented in a different random order). Each presentation consisted of a $500 \mathrm{~ms}$ fixation cross, an image flashed for $350 \mathrm{~ms}$, and then a $1300 \mathrm{~ms}$ response period (blank gray screen). The total number of timepoints was 1,224 (306 per run). Timepoints were classified as "resting" if they occured more than 4 seconds after the end of one stimulus block and less than 4 seconds after the start of the next stimulus block.

2.2.5. Functional Region of Interest Definition-Regressors for faces, scenes, objects, and scrambled objects in the localizer experiment were constructed by using the standard block hemodynamic model in AFNI (Cox 1996). LOC, PPA, RSC, and TOS were defined using the following contrasts: LOC, top 500 voxels for Objects > Scrambled near lateral occipital surface; PPA, top 300 voxels for Scenes > Objects near parahippocampal gyrus; RSC, top 200 voxels for Scenes > Objects near retrosplenial cortex; TOS, top 200 voxels for Scenes > Objects near the transverse occipital sulcus. The volume of each ROI in $\mathrm{mm}^{3}$ was chosen conservatively, based on previous results (Golarai, et al., 2007). Consistent with the metaanalysis by Nasr et al. (2011), PPA in our subjects was found to be centered on the collateral sulcus adjacent to the parahippocampal gyrus.

\subsection{Scene Category Experiment}

2.3.1. Participants -8 subjects ( 4 female) with normal or corrected-to-normal vision participated in the scene category fMRI experiment (these subjects did not overlap with those in the object-in-scene experiment). The study protocol was approved by the University of Illinois Institutional Review Board, and all subjects gave their written informed consent.

2.3.2. Scanning Parameters-Functional imaging data were acquired with a 3 Tesla Siemens Trio scanner. A gradient echo, echo-planar sequence was used to obtain functional images [volume repetition time (TR), $1.75 \mathrm{~s}$; echo time (TE), $30 \mathrm{~ms}$; flip angle, $90^{\circ}$; matrix, $64 \times 64$ voxels; FOV, $19 \mathrm{~cm}$; 29 oblique $3 \mathrm{~mm}$ slices with $0 \mathrm{~mm}$ gap; in-plane resolution, $3.0 \times 3.0 \mathrm{~mm}]$. The functional data was motion-corrected and each voxel's mean value was scaled to equal 100 (no spatial smoothing was applied). We collected a high-resolution structural scan for each subject; 4 subjects were scanned in a 3 Tesla Siemens Trio scanner (MPRAGE; $1 \times 1 \times 1.2 \mathrm{~mm}$, TR, $1900 \mathrm{~ms}$; TE, $2.25 \mathrm{~ms}$, flip angle, $9^{\circ}$ ) and 4 subjects were scanned in a 3 Tesla Siemens Allegra (MPRAGE; $1.25 \times 1.25 \times 1.25 \mathrm{~mm}$, TR, $2000 \mathrm{~ms}$; TE, $2.22 \mathrm{~ms}$, flip angle, $8^{\circ}$ ). The structural scan was used to calculate a transformation between each subject's brain and the Talairach atlas.

2.3.3. Stimuli and Procedure-Images $(800 \times 600$ pixels; subtending $24 \times 18$ degrees of visual angle) were presented in the center of the display using a back-projection system (Resonance Technologies) operating at a resolution of $800 \times 600$ pixels at $60 \mathrm{~Hz}$. For each run, subjects were instructed to count the number of images belonging to a target category (beaches, cities, highways or mountains; see example stimuli in Fig. 1c). On average, there 
were 16 target images perrun, ranging from 15-17 targets. Stimuli were presented in blocks of 8 images with a display time of $1.75 \mathrm{~s}$ for each image. Images within a block were primarily from the same natural scene category; however, in order to increase the difficulty of the counting task, one or two outgroup images from different scene categories (intrusions) occasionally appeared within a block. A fixation cross was presented throughout each block, and subjects were instructed to maintain fixation. There were 8 blocks in each run ( 2 blocks for each natural scene category), interleaved with $12 \mathrm{~s}$ fixation periods to allow for the hemodynamic response to return to baseline levels. A session contained 16 such runs, and the order of categories and intrusion images were counterbalanced and randomized across blocks. The total number of timepoints was 2,064 (129 per run). Timepoints were classified as "resting" if they occured more than 4 seconds after the end of one stimulus block and less than 4 seconds after the start of the next stimulus block.

2.3.4. Functional Region of Interest Definition-ROIs were defined using an independent localizer scan, consisting of blocks of face, object, scrambled object, landscape, and cityscape images. Each block consisted of 20 images presented for $450 \mathrm{~ms}$ each with a $330 \mathrm{~ms}$ interstimulus interval. Each of the five types of stimuli was presented four times during a run, with $12 \mathrm{~s}$ fixation periods after two or three blocks. Subjects completed two runs, performing a one-back task during the localizer by pressing a button every time an image was repeated. Regressors for faces, scenes, objects, and scrambled objects were constructed by using the standard block hemodynamic model in AFNI (Cox 1996), and the following contrasts were used to define ROIs: LOC, Objects > Scrambled near lateral occipital surface; PPA, Scenes > Objects near parahippocampal gyrus; RSC, Scenes > Objects near retrosplenial cortex; TOS, Scenes $>$ Objects near the transverse occipital sulcus. A threshold of $p<2 \cdot 10^{-3}$ (uncorrected) was applied, and was tightened to break clusters if necessary.

\subsection{Caudal IPL Definition}

Caudal IPL is a region strongly connected to macaque parahippocampal cortex (Kravitz et al. 2011b) for which we do not have a functional localizer. In order to evaluate the match between the macaque and human connectivity patterns, we sought to anatomically define a human region equivalent to cIPL. The two caudal-most areas of human IPL (defined using probabilistic cytoarchitectonic maps) are PGa and PGp, which are thought to correspond to the caudal-most sections of macaque IPL, PG and Opt (Caspers et al. 2011). Of these, PGp exhibits significantly stronger functional and structural connectivity with the parahippocampal gyrus (Uddin et al. 2010), giving the best match with the proposed parietomedial temporal pathway targeting parahippocampal areas from cIPL. We therefore define cIPL in all subjects using the Eickhoff-Zilles PGp probabalistic cytoarchitectonic map (Eickhoff et al. 2005, based on Caspers et al. 2006, 2008). We thresholded the map at $p$ > 0.5 , and transformed the map into each subject's native space. Since cIPL slightly overlapped TOS in some subjects, any voxels shared between cIPL and TOS were excluded from both regions (no other ROIs included overlapping voxels).

\subsection{PPA Connectivity Analysis: ROIs}

We first learned PPA connectivity maps for four pre-defined seed regions: lateral occipital complex (LOC), transverse occipital sulcus (TOS, also referred to as the "occipital place area" in Dilks et al. 2013), retrosplenial cortex (RSC), and caudal inferior parietal lobule (cIPL) by setting $A^{1}$ to be PPA and $A^{2}$ to be one of the four seed regions. To avoid functional connectivity idiosyncratic to a specific experiment or task, we used data from both the object-in-scene experiment and the scene category experiment (see above). 
We first validated that our method could learn meaningful voxel-level connectivity maps which provide better generalization performance, compared to a connectivity map which is constant over left PPA and constant over right PPA. For each seed region and subject, we learned a connectivity map using one training run, and tuned the smoothness parameter $\lambda$ to maximize the fraction of variance explained on a validation set consisting of all but one of the remaining runs. The classifier was then retrained on both the training run and validation set (using the selected $\lambda$ value) and tested on the final held-out testing run. Results were averaged across all choices of training run, with a random testing run being chosen for each training run. These results were compared to those from ROI-level connectivity maps, in which all PPA voxels in each hemisphere were constrained to take on the same value (equivalent to $\lambda \rightarrow \infty$ ).

We then learned a weightmap over PPA for each subject and for each seed region using all experimental runs, with $\lambda$ chosen such that the average fraction of variance explained, when training on one run and testing on the other runs, was maximized. We measured the correlation between the connectivity weights and the anterior-posterior voxel coordinates, to obtain a simple measure of how the learned weights in PPA varied along the anteriorposterior axis. The correlation was computed separately for left and right PPA (except where specified, results below are collapsed across left and right PPA).

\subsection{PPA Connectivity Analysis: Whole-Brain}

To explore the connectivity patterns between PPA and the rest of the brain, we performed a whole-brain searchlight connectivity analysis in which our seed region was densely sampled throughout the entire cortex. We fixed $A^{1}$ to be PPA, and then placed a $3 \times 3 \times 3$ voxel searchlight $A^{2}$ at each point on a lattice with 2 voxel spacing. For each searchlight, we used all experimental runs to learn a map of connectivity weights in PPA, and then measured the correlation between the learned weights and the anterior-posterior axis. We obtained an anterior PPA vs. posterior PPA preference for each brain voxel by averaging the correlation value of all searchlights which included that voxel. In order to speed up computation, we used a single value of $\lambda=5.5$ for all subjects, equal to the average of the optimal $\lambda$ values in the ROI experiment (in log space). Group-level statistics were computed by transforming each subject's results into Talairach space.

\subsection{Scene- and Object-Sensitivity Analysis}

After identifying connectivity differences among PPA voxels, we investigated whether these connectivity gradients corresponded to functional differences in stimulus selectivity. To measure the response properties of individual PPA voxels, we examined the statistics from the regressors in the localizer experiment. For each voxel, the t-statistics from the scene and object regressors were recorded, and each voxel was also given a binary label of "significantly activated" or "not significantly activated" based on whether its false discovery rate (FDR) for each category was less than or greater than 0.05 . To detect a sensitivity gradient across PPA, the correlation between the anterior-posterior axis and the t-statistics was computed. For visualization purposes, each subject's PPA voxels were binned into 10 bins running anterior-posterior, and the mean t-statistic and percentage of activated voxels was calcuated for each bin, to give a sensitivity profile.

\subsection{LOC/TOS vs. RSC/cIPL Connectivity}

After discovering that LOC/TOS and RSC/cIPL connect preferentially to different voxels in PPA (see Results), we sought to place these connectivity gradients in the context of the entire parahippocampal region. For each cortical voxel, we averaged the coefficients for the voxel's correlations with LOC and TOS, and compared it to the average of the coefficients for the voxel's correlations with RSC and cIPL. We transformed each subject's correlation 
maps into Talairach space, and identified voxels at the group level that showed a consistent different across subjects to LOC/TOS functional connectivity vs. RSC/cIPL functional connectivity. In addition to the parahippocampal region, we searched all of cortex for voxels with this connectivity pattern.

\section{Results}

Since we are interested in the intrinsic connectivity properties of PPA (rather than functional correlations idiosyncratic to a specific stimulus set), we localized PPA in two separate groups of subjects, each of which then performed a different experimental task with different stimuli. Although both experiments included scenes, in one case (identifying scene category) the scenes were directly relevant to the task, while in the other (locating a target object in scenes) scenes were not the primary focus. Given these datasets, do we see connectivity differences in anterior versus posterior PPA analogous to those in macaque parahippocampal cortex? Note that, since the connectivity patterns were similar in both datasets (see Supplementary Fig. 4), all connectivity results below are collapsed across both experiments.

\subsection{PPA Connectivity Analysis: ROls}

We began our investigation of PPA's connectivity structure by learning PPA connectivity maps for four seed regions: two other scene-sensitive regions (TOS and RSC), an objectsensitive area in ventral occipital cortex (LOC), and a posterior parietal region known to exhibit parahippocampal connectivity (cIPL). We first confirmed that, for each individual subject, we could learn weight maps over PPA (describing its connectivity with each of these regions) that generalize well across runs. As shown in Fig. 2a, spatially smooth voxellevel connectivity maps in PPA predict activity in LOC, TOS, RSC, or cIPL better than a map which has only a single weight for left PPA and a single weight for right PPA (LOC: $t_{17}=4.42$; TOS: $t_{17}=4.63$; RSC: $t_{17}=7.80$; cIPL: $t_{17}=3.28$; all $p<0.01$, two-tailed paired t-test). These results were computed by choosing $\lambda$ to maximize the fraction of variance explained (on an independent validation set) but improvement over the traditional constantweight connectivity held for a wide range of regularization strengths $\lambda$ (see Supplementary Fig. 2). Although all regions showed at least some activity related to PPA's timecourse, a significantly smaller amount of the cIPL timecourse can be predicted by PPA (ROI-level: LOC >cIPL: $t_{17}=7.31$; TOS >cIPL: $t_{17}=10.58$; RSC >cIPL: $t_{17}=10.23$; Voxel-level: LOC >cIPL: $t_{17}=6.58$; TOS>cIPL: $t_{17}=12.12$; RSC >cIPL: $t_{17}=11.81$; all $p<0.01$ twotailed paired t-test), consistent with its proposed role as a general processing hub in parietal cortex with connections to many regions besides PPA (Caspers et al. 2011; Caspers et al. 2012)

Since meaningful voxel-level weight maps can be learned for individual subjects, we can ask whether these weight maps show any anterior-posterior differences which are consistent across subjects. If PPA shows the same gradient of connectivity as TH/TF/TFO, we expect the posterior portion of PPA to be more strongly connected to occipital visual regions LOC and TOS, with the anterior portion of PPA more strongly connected to RSC and cIPL. As shown in Fig. 2b, this is precisely what we observed; LOC and TOS connectivity weights tend to increase moving anterior to posterior, while RSC and cIPL weights increase in the opposite direction (LOC: $t_{17}=3.10, p<0.01$; TOS: $t_{17}=2.72, p=0.01$; RSC: $t_{17}=-3.76, p$ $<0.01$; cIPL: $t_{17}=-3.24, p<0.01$; two-tailed t-test after z-transform). These results are collapsed across left and right PPA; both hemispheres showed similar connectivity patterns, though effects were somewhat stronger in left PPA, by an average of $0.13\left(t_{17}=2.20, p=\right.$ 0.042; two-tailed t-test after z-transform). We did not observe significant differences along the inferior-superior axis or medial-lateral axis, except for preferential connectivity of cIPL to medial PPA (see Supplementary Fig. 3). 


\subsection{PPA Connectivity Analysis: Whole-Brain}

Having established a consistent posterior-anterior gradient of connectivity between our regions of interest and PPA, we then performed a searchlight analysis to search for other brain regions with posterior-anterior PPA connectivity gradients; rather than using our fixed ROIs as seed regions, we swept a $3 \times 3 \times 3$ voxel searchlight throughout the entire cortex. As in Fig. 2b, we learn a PPA connectivity map for each seed region and compute the correlation of this map with the anterior-posterior axis; those seed regions which induce a PPA weight map that is positively correlated with the anterior-posterior axis are preferentially connected to posterior PPA, while those inducing a negatively correlated weight map are preferentially connected to anterior PPA. The traditional (homogeneous) model of PPA predicts that consistent preferential connectivity should only occur for seed regions directly adjacent to posterior or anterior PPA (which will be correlated with the nearer part of PPA due to local noise). If PPA contains subregions similar to those in macaque, however, we would expect a number of regions throughout cortex to show preferential connectivity patterns which are both consistently non-zero and in opposite directions.

Our results are shown in Fig. 3. As predicted by our subregion hypothesis, seed regions in occipital visual areas (including LOC and TOS) showed preferential connectivity to posterior PPA, while RSC and cIPL showed preferential connectivity to anterior PPA. Note that these results cannot be explained by local noise correlations, since RSC and cIPL are physically closer to the posterior edge of PPA. We also observed connectivity to anterior PPA in ventral prefrontal cortex (PFC) (primarily on the medial surface) and on the lateral surface of the anterior temporal lobe. Regions immediately anterior to PPA, including the hippocampus and anterior parahippocampal gyus, show preferential correlation with anterior PPA, but it is unclear if this effect is driven by intrinsic connectivity or local noise correlations. Coronal and axial slices are shown in Fig. 4, demonstrating that these connectivity patterns are bilaterally symmetric. This result can also be obtained by using only "resting" timepoints from between stimulus blocks or using a different value for $\lambda$, and is apparent for both the scene and object tasks (see Supplementary Fig. 4), suggesting that this connectivity pattern is intrinsic rather than task-specific. The fraction of variance explained for the searchlights is consistent with our ROI analysis, showing the strongest coupling between PPA and visual regions including LOC, TOS, and RSC (see Supplementary Fig. 5).

\subsection{Scene- and Object-Sensitivity Analysis}

Do these connectivity differences give rise to differences in functional response to stimulus categories? Although the functional roles of anterior and posterior PPA are likely complex, a simple functional anterior-posterior distinction can be seen in the scene and object responses during our localizer experiment. The selectivities of the PPA voxels to scenes and objects are shown in Fig. 5, binned based on position along the anterior-posterior axis. At the posterior side of PPA, the sensitivity to both scenes and objects is high, with nearly all voxels responding to scene stimuli and a majority of voxels responding to object stimuli. Moving posterior to anterior, scene selectivity decreases somewhat (average correlation between t-statistic and posterior-anterior axis of $0.25, t_{10}=3.00, p=0.01$ two-tailed t-test after z-transform), although most voxels respond significantly to scene stimuli across all of PPA. Object sensitivity, however, substantially decreases (average correlation between tstatistic and posterior-anterior axis of $0.32, t_{10}=3.39, p<0.01$ two-tailed t-test after $\mathrm{z}$ transform), with a majority of voxels at the anterior edge showing no significant response to object stimuli. 


\subsection{LOC/TOS vs. RSC/cIPL Connectivity}

A number of studies have examined functional and connectivity gradients along the entire parahippocampal gyrus, which includes (in addition to PPA) a portion of parahippocampal cortex anterior to PPA, and the perirhinal cortex (Aminoff et al. 2007; Bar and Aminoff 2003; Litman et al. 2009; Staresina et al. 2011; Libby et al. 2012). In order to examine how our gradients within PPA fit into the connectivity patterns of the broader medial temporal lobe, we searched for voxels which showed the same connectivity differences we observed within PPA. As shown in Fig. 6, the pattern of connectivity observed in anterior PPA (RSC and cIPL greater than LOC and TOS) extends anteriorly along the parahippocampal gyrus and into the hippocampus. The most anterior portion of the parahippocampal gyrus (around perirhinal cortex) did not show a connectivity pattern matching either anterior or posterior PPA, consistent with previous work on the connectivity properties of perirhinal cortex (Libby et al. 2012). In general, the regions showing connectivity similar to anterior PPA overlap very well with the Default Mode Network (Fox et al. 2005) (see Supplementary Fig. $6)$.

\section{Discussion}

Our results demonstrate that human PPA exhibits a gradient in connectivity along the anterior-posterior axis analogous to the gradient in connectivity along macaque TH/TF/TFO. This connectivity gradient was also paired with a functional gradient of sensitivity to scene and abstract object stimuli. These results present a challenge to current models of PPA function which assume that PPA is functionally homogeneous, and demonstrate that anterior and posterior PPA connect differentially to two distinct cortical networks.

Note that, although our data suggest that PPA might contain identifiable subregions, these subregions should not be considered as completely independent modules. Both subregions activate selectively to scenes, and the parahippocampal region (at least in macaques) is densely self-connected (Suzuki 2009), implying that these subregions cooperate to build a complete representation of a scene. Their distinct connectivity properties, however, do suggest that each may be involved in specific aspects of visual and cognitive processing involved in the overarching goal of scene understanding. We discuss some possibilities for the functional roles of the subregions below.

\subsection{Posterior PPA}

Posterior PPA shows a stronger response to abstract objects than anterior PPA, and is more strongly connected to all of occipital visual cortex, including LOC and TOS. These regions have well-defined retinotopic maps (Arcaro et al. 2009; Nasr et al. 2011), and are associated with the perception of low-level visual features and object shape. Previous work has hinted that posterior PPA is more responsive to both simple visual textures and objects; Arcaro et al. (2009) found that a posterior portion of PPA responded about four times as strongly to a flickering checkerboard stimulus compared to an anterior portion, and that the response to objects was greater than the response to scrambled images only in the posterior portion. In other words, posterior PPA may be more visually responsive than anterior PPA.

Posterior PPA may be specifically tuned to visual features in the high spatial frequency band; PPA has been shown to respond preferentially to higher spatial frequencies, and this effect tended to be strongest at the posterior end of PPA (Rajimehr et al. 2011). Highfrequency edges could be the most important visual features for understanding the structure of a scene and navigating through it (Rajimehr et al. 2011, Walther et al. 2011).

Alternatively, this high-frequency preference could be related to the perception of large, landmark-like objects. A comparison of the Fourier spectra of 400 objects with either large 
or small real-world size (but matched visual size) found that larger objects tend to have more power at high spatial frequencies, especially along horizontal and vertical orientations; intuitively, larger objects are "boxier" while smaller objects are rounder (Konkle 2011). A region of cortex selective for large objects has been shown to overlap with about half of PPA near the collateral sulcus, possibly corresponding to posterior PPA (Konkle and Oliva 2012).

It is also possible that posterior PPA performs texture and ensemble processing, since these tasks tend to activate cortical regions around the collateral sulcus, overlapping with posterior PPA (Cant and Goodale 2007; Cant and Goodale 2011; Cant and Xu 2012). Although Cant and $\mathrm{Xu}$ (2012) failed to find an anterior-posterior difference within PPA for ensemble or texture processing by splitting PPA along the center of activation, a more sensitive voxellevel measure could potentially reveal such a gradient.

Our description of posterior PPA is in fact similar to the original proposal of Aguirre et al. for a "lingual landmark area" (LLA), slightly posterior to PPA, which was "specialized for the perception of visual stimuli with orienting value" (Aguirre et al. 1998) and carried out bottom-up perceptual analysis to recognize locations or landmarks (Epstein et al. 1999; Cant and Goodale 2007). Although the LLA is no longer identified as an independent region from PPA in current studies, it is possible that the posterior portion of PPA corresponds to the properties of the proposed LLA, offering an explanation for why this region was localized more posteriorly than the full PPA.

\subsection{Anterior PPA}

Anterior PPA is specifically connected to RSC, cIPL, medial PFC, and the lateral surface of the anterior temporal lobe. In addition, this portion of PPA is less visually responsive to both scenes and objects, with notably low sensitivity to abstract object stimuli.

The set of regions connected more strongly to anterior PPA is strikingly similar to the Default Mode Network (DMN) (Raichle et al. 2001; Fox et al. 2005; Buckner et al. 2008), which is known to include the parahippocampal region. The only portions of the DMN that do not show differential connectivity to anterior PPA in our data are the rostral portion of the posterior cingulate cortex (PCC) and the superior frontal cortex. It is likely that PPA does not have direct connections to these regions; a DTI study showed that the medial temporal lobe directly connects only to RSC, rather than more rostral PCC (Greicius et al. 2009), and a functional connectivity analysis showed that the hippocampal formation (including the parahippocampal region) is connected only to the more ventral portion of the prefrontal cortex, not the dorsal portion (Buckner et al. 2008). We confirmed that RSC and cIPL showed connectivity with the entire DMN (see Supplementary Fig. 6), suggesting that anterior PPA does have indirect connections to PCC and superior frontal cortex.

Although the DMN has been implicated in a large number of internally-focused tasks, one of its key roles involves autobiographical memory (Buckner et al. 2008). Models of recognition memory have previously identified parahippocampal cortex as primarily encoding spatial context information (Eichenbaum et al. 2007), and data from Aminoff et al. (2007) has suggested that an anterior portion of PPA may be involved in recall based on spatial context. Our results are consistent with anterior PPA playing a more central role in memory than posterior PPA, given anterior PPA's connectivity with the DMN.

PPA is known to represent global scene properties such as spatial expanse (Park et al. 2011, Kravitz et al. 2011a), and to construct global scene representations which are not predictable from responses to signature objects (MacEvoy and Epstein 2011). Anterior PPA's connectivity to cIPL and RSC, along with its lower sensitivity to abstract objects, suggest 
that it may be more concerned with these types of spatial and non-object-based scene properties than posterior PPA. Future research contrasting global and object-based properties of scenes, however, would be necessary to test such a hypothesis.

The fact that anterior PPA had a lower sensitivity to our abstract object stimuli does not necessarily imply that this region does not use object information. Previous work has shown that PPA responds to objects that have spatial associations (Aminoff et al. 2007), are spacedefining (Mullally and Maguire 2011), and are navigationally-relevant (Janzen and Van Turennout 2004). These types of responses require spatial memory and cannot be based purely on visual features like object shape. If anterior PPA is involved in processing spatial context, then space-defining or navigationally-relevant objects could activate anterior PPA more strongly than our abstract objects, which were unfamiliar and provided no sense of context or orientation. Further experiments will be required to determine whether what type of object-related information is used in this region.

\subsection{Homology with TH/TF/TFO}

Given the close match between the connectivity gradients in macaque parahippocampal cortex and those in PPA, can we identify a precise correspondence between macaque regions TH/TF/TFO and our PPA subregions? Since the connectivity gradients extend anteriorly beyond PPA (which terminates in the most posterior part of the parahippocampal gyrus), a possible homology could identify posterior PPA with TFO, anterior PPA with TF, and the anterior portion of parahippocampal cortex with TH. This labeling would be consistent with previous work showing that TFO is more visually responsive than TF and may have a coarse retinotopy (Saleem et al. 2007), matching the properties of posterior PPA. This correspondence will only be definitively confirmed, however, if future electrophysiological measurements show that TH does not respond to scene stimuli (placing it anterior to anterior PPA) while TF and TFO do.

\subsection{Implications for Future Work on PPA}

Unraveling the functions of the PPA has proven to be a challenging problem, given the region's involvement in a variety of scene perception and navigation tasks (Epstein 2008). Our results imply that a complete model of PPA's functional properties must account for the differences in connectivity and function between anterior and posterior PPA. Although the precise roles of PPA's subregions are yet to be determined, our results and previous work suggest that posterior PPA is concerned primarily with perception of low-level visual features and object shape, while anterior PPA is involved in memory and global contextual processing. Given the relatively small size of each of these subregions, voxel-level approaches (such as our connectivity method) as well as high-resolution fMRI imaging may be required to identify the representations evoked within the parts of PPA, and understand how these regions cooperate to build a coherent scene representation.

\section{Conclusions}

Our connectivity findings call into question the traditional view of PPA as a homogeneous region performing a single functional role, and provide a starting point for future experimental and modeling work investigating how different types of cortical networks interact for scene understanding and recognition. This discovery was made possible by our voxel-level functional connectivity approach, which may prove fruitful for uncovering subregions in other cortical systems. 


\section{Supplementary Material}

Refer to Web version on PubMed Central for supplementary material.

\section{Acknowledgments}

We thank the Richard M. Lucas Center for Imaging, two anonymous reviewers, and Audrey Lustig (for the data from the Scene Category experiment). This work is funded by National Institutes of Health Grant 1 R01 EY019429 (to L.F.-F. and D.M.B.) and a National Science Foundation Graduate Research Fellowship under Grant No. DGE-0645962 (to C.B.).

\section{References}

Aguirre GK, Zarahn E, DEsposito M. An area within human ventral cortex sensitive to building stimuli: evidence and implications. Neuron. 1998; 21:373-383. [PubMed: 9728918]

Aminoff E, Gronau N, Bar M. The parahippocampal cortex mediates spatial and nonspatial associations. Cereb. Cortex. 2007; 17:1493-1503. [PubMed: 16990438]

Arcaro M, McMains S, Singer B, Kastner S. Retinotopic organization of human ventral visual cortex. J. Neurosci. 2009; 29:10638-10652. [PubMed: 19710316]

Baldassano C, Iordan MC, Beck DM, Fei-Fei L. Voxel-level functional connectivity using spatial regularization. Neuroimage. 2012; 63:1099-1106. [PubMed: 22846660]

Bar M. Visual objects in context. Nat. Rev. Neurosci. 2004; 5:617-629. [PubMed: 15263892]

Bar M, Aminoff E. Cortical analysis of visual context. Neuron. 2003; 38:347-358. [PubMed: 12718867]

Buckner RL, Andrews-Hanna JR, Schacter DL. The brains default network: anatomy, function, and relevance to disease. Annals Of The New York Academy Of Sciences. 2008; 1124:1-38. [PubMed: 18400922]

Cant JS, Goodale MA. Attention to form or surface properties modulates different regions of human occipitotemporal cortex. Cerebral Cortex. 2007; 17:713-731. [PubMed: 16648452]

Cant JS, Goodale MA. Scratching beneath the surface: new insights into the functional properties of the lateral occipital area and parahippocampal place area. Journal of Neuroscience. 2011; 31:82488258. [PubMed: 21632946]

Cant JS, Xu Y. Object ensemble processing in human anterior-medial ventral visual cortex. Journal of Neuroscience. 2012; 32:7685-7700. [PubMed: 22649247]

Caspers S, Eickhoff SB, Geyer S, Scheperjans F, Mohlberg H, Zilles K, Amunts K. The human inferior parietal lobule in stereotaxic space. Brain structure function. 2008; 212:481-495. [PubMed: 18651173]

Caspers S, Eickhoff SB, Rick T, Von Kapri A, Kuhlen T, Huang R, Shah NJ, Zilles K. Probabilistic fibre tract analysis of cytoarchi-tectonically defined human inferior parietal lobule areas reveals similarities to macaques. NeuroImage. 2011; 58:362-380. [PubMed: 21718787]

Caspers S, Geyer S, Schleicher A, Mohlberg H, Amunts K, Zilles K. The human inferior parietal cortex: cytoarchitectonic parcellation and interindividual variability. NeuroImage. 2006; 33:430448. [PubMed: 16949304]

Caspers S, Schleicher A, Bacha-Trams M, Palomero-Gallagher N, Amunts K, Zilles K. Organization of the human inferior parietal lobule based on receptor architectonics. Cerebral Cortex. 2012:1-14.

Cavada C, Goldman-Rakic PS. Posterior parietal cortex in rhesus monkey: I. Parcellation of areas based on distinctive limbic and sensory corticocortical connections. J. Comp. Neurol. 1989; 287:393-421. [PubMed: 2477405]

Chai B, Walther D, Beck D, Fei-Fei L. Exploring functional connectivity of the human brain using multivariate information analysis. NIPS. 2009; 22

Cohen AL, Fair DA, Dosenbach NU, Miezin FM, Dierker D, Van Essen DC, Schlaggar BL, Petersen SE. Defining functional areas in individual human brains using resting functional connectivity MRI. Neuroimage. 2008; 41:45-57. [PubMed: 18367410] 
Cox RW. AFNI: software for analysis and visualization of functional magnetic resonance neuroimages. Comput. Biomed. Res. 1996; 29:162-173. [PubMed: 8812068]

Dilks DD, Julian JB, Paunov AM, Kanwisher N. The occipital place area is causally and selectively involved in scene perception. J. Neurosci. 2013; 33:1331-1336. [PubMed: 23345209]

Eichenbaum H, Yonelinas AP, Ranganath C. The medial temporal lobe and recognition memory. Annu. Rev. Neurosci. 2007; 30:123-152. [PubMed: 17417939]

Eickhoff SB, Stephan KE, Mohlberg H, Grefkes C, Fink GR, Amunts K, Zilles K. A new spm toolbox for combining probabilistic cytoarchitectonic maps and functional imaging data. NeuroImage. 2005; 25:1325-1335. [PubMed: 15850749]

Epstein R. Parahippocampal and retrosplenial contributions to human spatial navigation. Trends in Cognitive Sciences. 2008; 12:388-396. [PubMed: 18760955]

Epstein R, Graham KS, Downing PE. Viewpoint-specific scene representations in human parahippocampal cortex. Neuron. 2003; 37:865-876. [PubMed: 12628176]

Epstein R, Harris A, Stanley D, Kanwisher N. The parahip-pocampal place area: recognition, navigation, or encoding? Neuron. 1999; 23:115-125. [PubMed: 10402198]

Epstein R, Kanwisher N. A cortical representation of the local visual environment. Nature. 1998; 392:598-601. [PubMed: 9560155]

Epstein RA, Morgan LK. Neural responses to visual scenes reveals inconsistencies between fMRI adaptation and multivoxel pattern analysis. Neuropsychologia. 2012; 50:530-543. [PubMed: 22001314]

Fox MD, Snyder AZ, Vincent JL, Corbetta M, Van Essen DC, Raichle ME. The human brain is intrinsically organized into dynamic, anticorrelated functional networks. Proc. Natl. Acad. Sci. U.S.A. 2005; 102:9673-9678. [PubMed: 15976020]

Golarai G, Ghahremani DG, Whitfield-Gabrieli S, Reiss A, Eberhardt JL, Gabrieli JD, Grill-Spector K. Differential development of high-level visual cortex correlates with category-specific recognition memory. Nat. Neurosci. 2007; 10:512-522. [PubMed: 17351637]

Grant, M.; Boyd, S. CVX: Matlab software for disciplined convex programming, version 1.21. 2011. http://cvxr.com/cvx

Greicius MD, Supekar K, Menon V, Dougherty RF. Resting-state functional connectivity reflects structural connectivity in the default mode network. Cerebral Cortex. 2009; 19:72-78. [PubMed: 18403396]

Grill-Spector K, Kushnir T, Edelman S, Avidan G, Itzchak Y, Malach R. Differential processing of objects under various viewing conditions in the human lateral occipital complex. Neuron. 1999; 24:187-203. [PubMed: 10677037]

Harel A, Kravitz DJ, Baker CI. Deconstructing Visual Scenes in Cortex: Gradients of Object and Spatial Layout Information. Cereb Cortex. 2012

Heinzle J, Kahnt T, Haynes J. Topographically specific functional connectivity between visual field maps in the human brain. NeuroImage. 2011; 56:1426-1436. [PubMed: 21376818]

Janzen G, Van Turennout M. Selective neural representation of objects relevant for navigation. Nat Neurosci. 2004; 7:673-677. [PubMed: 15146191]

Kahn I, Andrews-Hanna JR, Vincent JL, Snyder AZ, Buckner RL. Distinct cortical anatomy linked to subregions of the medial temporal lobe revealed by intrinsic functional connectivity. Journal of Neurophysiology. 2008; 100:129-139. [PubMed: 18385483]

Kim DS, Kim M. Combining functional and diffusion tensor mri. Annals of the New York Academy of Sciences. 2005; 1064:1-15. [PubMed: 16394144]

Kim JH, Lee JM, Jo HJ, Kim SH, Lee JH, Kim ST, Seo SW, Cox RW, Na DL, Kim SI, Saad ZS. Defining functional SMA and pre-SMA subregions in human MFC using resting state fMRI: functional connectivity-based parcellation method. Neuroimage. 2010; 49:2375-2386. [PubMed: 19837176]

Konkle, T. Ph.D. thesis. Cambridge, MA: Massachusetts Institute of Technology; 2011. The role of real-world size in object representation.

Konkle T, Oliva A. A real-world size organization of object responses in occipitotemporal cortex. Neuron. 2012; 74:1114-1124. [PubMed: 22726840] 
Kravitz DJ, Peng CS, Baker CI. Real-world scene representations in high-level visual cortex: it's the spaces more than the places. J. Neurosci. 2011a; 31:7322-7333.

Kravitz DJ, Saleem KS, Baker CI, Mishkin M. A new neural framework for visuospatial processing. Nat. Rev. Neurosci. 2011b; 12:217-230. [PubMed: 21415848]

Libby LA, Ekstrom AD, Ragland JD, Ranganath C. Differential connectivity of perirhinal and parahippocampal cortices within human hippocampal subregions revealed by high-resolution functional imaging. Journal of Neuroscience. 2012; 32:6550-6560. [PubMed: 22573677]

Litman L, Awipi T, Davachi L. Category-specificity in the human medial temporal lobe cortex. Hippocampus. 2009; 19:308-319. [PubMed: 18988234]

MacEvoy SP, Epstein RA. Constructing scenes from objects in human occipitotemporal cortex. Nature Neuroscience. 2011; 14:1323-1329.

Margulies DS, Kelly AM, Uddin LQ, Biswal BB, Castellanos FX, Milham MP. Mapping the functional connectivity of anterior cingulate cortex. Neuroimage. 2007; 37:579-588. [PubMed: 17604651]

Margulies DS, Vincent JL, Kelly C, Lohmann G, Uddin LQ, Biswal BB, Villringer A, Castellanos FX, Milham MP, Petrides M. Precuneus shares intrinsic functional architecture in humans and monkeys. PNAS. 2009; 106:20069-20074. [PubMed: 19903877]

Mullally SL, Maguire EA. A new role for the parahippocampal cortex in representing space. J. Neurosci. 2011; 31:7441-7449. [PubMed: 21593327]

Nasr S, Liu N, Devaney KJ, Yue X, Rajimehr R, Ungerleider LG, Tootell RB. Scene-selective cortical regions in human and nonhuman primates. J. Neurosci. 2011; 31:13771-13785. [PubMed: 21957240]

Park S, Brady TF, Greene MR, Oliva A. Disentangling scene content from spatial boundary: complementary roles for the parahip-pocampal place area and lateral occipital complex in representing real-world scenes. J. Neurosci. 2011; 31:1333-1340. [PubMed: 21273418]

Raichle ME, MacLeod AM, Snyder AZ, Powers WJ, Gusnard DA, Shulman GL. A default mode of brain function. Proceedings of the National Academy of Sciences of the United States of America. 2001; 98:676-682. [PubMed: 11209064]

Rajimehr R, Devaney KJ, Bilenko NY, Young JC, Tootell RB. The "parahippocampal place area" responds preferentially to high spatial frequencies in humans and monkeys. PLoS Biol. 2011; 9:e1000608. [PubMed: 21483719]

Roy AK, Shehzad Z, Margulies DS, Kelly AM, Uddin LQ, Gotimer K, Biswal BB, Castellanos FX, Milham MP. Functional connectivity of the human amygdala using resting state fMRI. Neuroimage. 2009; 45:614-626. [PubMed: 19110061]

Rushworth MF, Behrens TE, Johansen-Berg H. Connection patterns distinguish 3 regions of human parietal cortex. Cereb. Cortex. 2006; 16:1418-1430.

Saleem KS, Price JL, Hashikawa T. Cytoarchitectonic and chemoarchitectonic subdivisions of the perirhinal and parahippocampal cortices in macaque monkeys. J. Comp. Neurol. 2007; 500:9731006. [PubMed: 17183540]

Sewards TV. Neural structures and mechanisms involved in scene recognition: a review and interpretation. Neuropsychologia. 2011; 49:277-298. [PubMed: 21095199]

Staresina BP, Duncan KD, Davachi L. Perirhinal and parahip-pocampal cortices differentially contribute to later recollection of object-and scene-related event details. Journal of Neuroscience. 2011; 31:8739-8747. [PubMed: 21677158]

Suzuki, WA. Comparative analysis of the cortical afferents, intrinsic projections and interconnections of the parahippocampal region in monkeys and rats. In: Gazzaniga, MS., editor. The Cognitive Neurosciences. The MIT Press; 2009. p. 659-674.

Uddin LQ, Supekar K, Amin H, Rykhlevskaia E, Nguyen DA, Greicius MD, Menon V. Dissociable connectivity within human angular gyrus and intraparietal sulcus: evidence from functional and structural connectivity. Cerebral Cortex. 2010; 20:2636-2646. [PubMed: 20154013]

Walther DB, Chai B, Caddigan E, Beck DM, Fei-Fei L. Simple line drawings suffice for functional mri decoding of natural scene categories. Proc. Nat. Acad. of Sci (PNAS). 2011 
Weiner KS, Grill-Spector K. Not one extrastriate body area: using anatomical landmarks, hMT+, and visual field maps to parcellate limb-selective activations in human lateral occipitotemporal cortex. Neuroimage. 2011; 56:2183-2199. [PubMed: 21439386]

Zhang D, Snyder AZ, Fox MD, Sansbury MW, Shimony JS, Raichle ME. Intrinsic functional relations between human cerebral cortex and thalamus. J. Neurophysiol. 2008; 100:1740-1748. [PubMed: 18701759] 


\section{Highlights}

Connectivity and functional properties vary within the Parahippocampal Place Area. Occipital visual regions such as LOC are better connected to posterior PPA.

The default mode network, including RSC, is better connected to anterior PPA. Abstract sculptures activate posterior PPA more strongly than anterior PPA. 

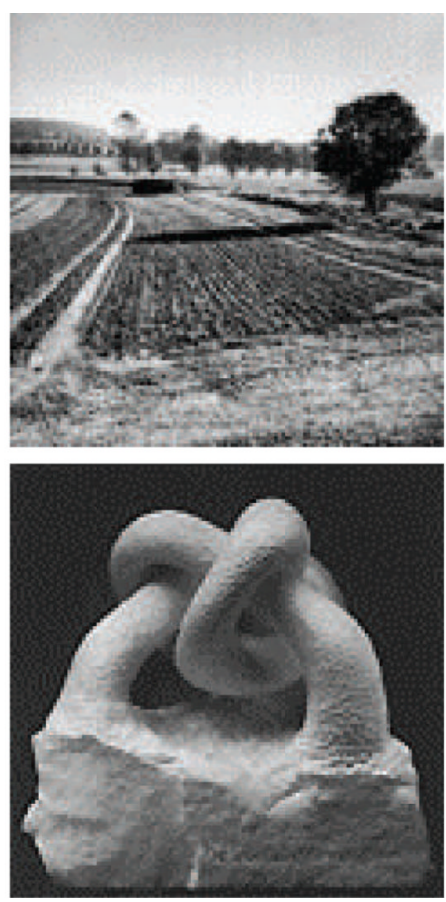

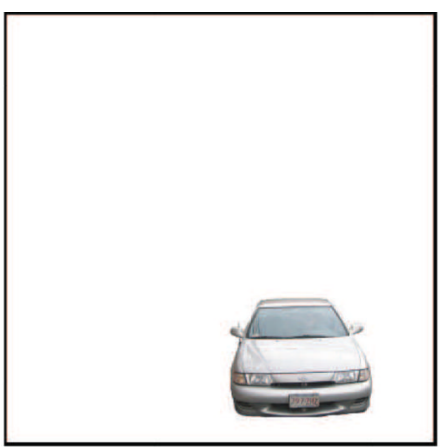

$\mathrm{b}$

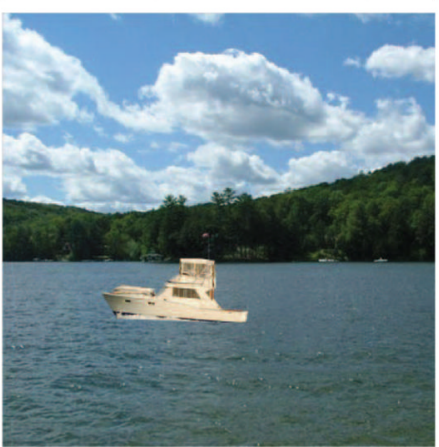

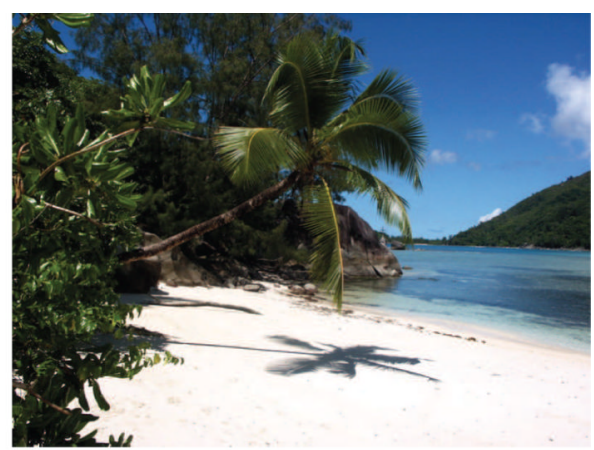

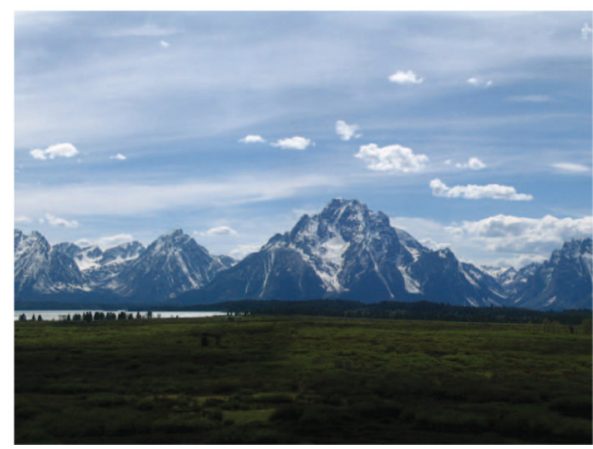

Figure 1. Sample stimuli used in our experiments

(a) Scene and object stimuli from the localizer experiment, which also included faces and scrambled objects. (b) Isolated object and object-in-scene stimuli from the object-in-scene experiment. (c) Beach and mountain stimuli from the scene category experiment, which also included cities and highways. 

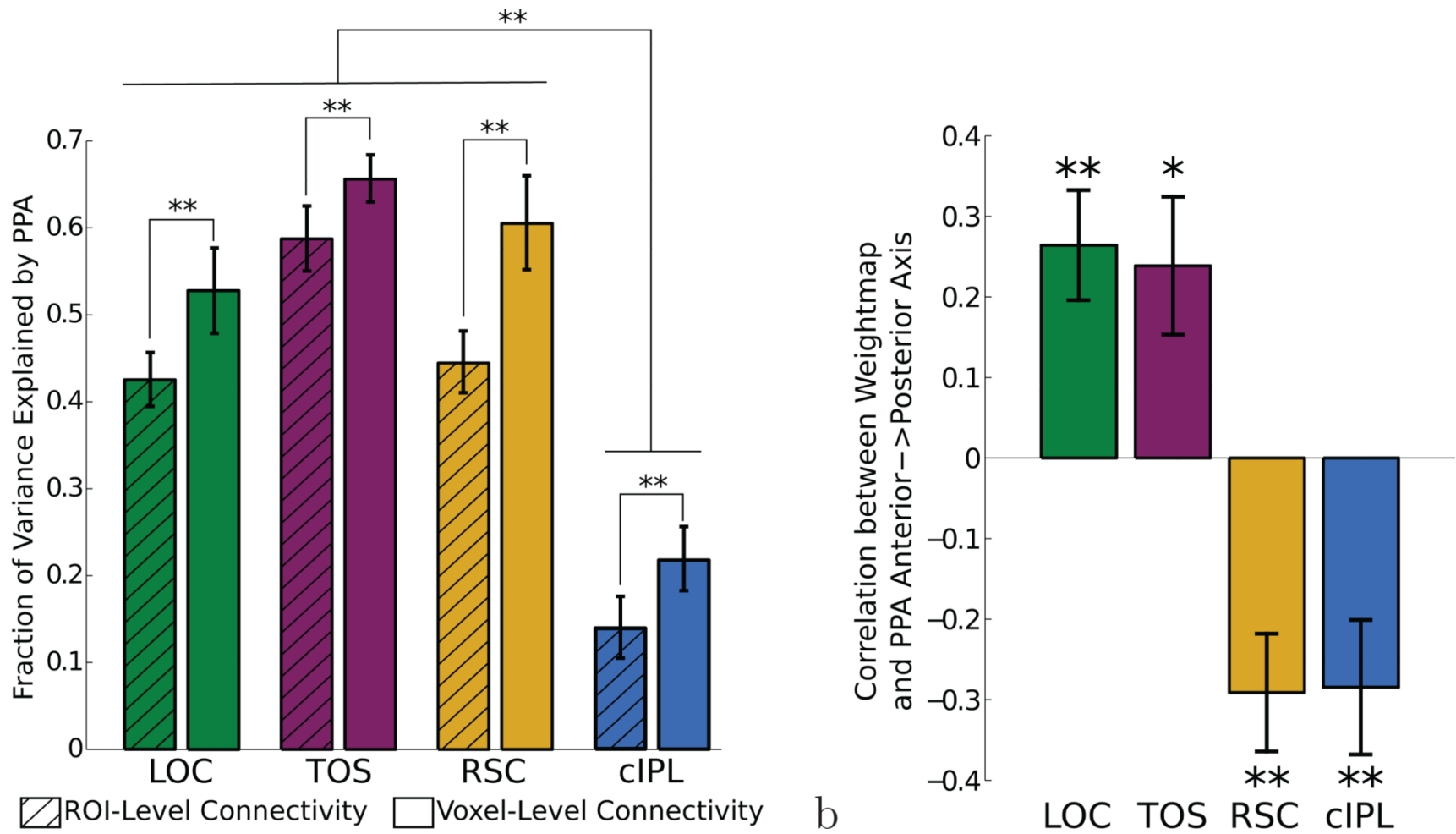

Figure 2. A comparison of the learned PPA weightmaps and the overall connectivity strength, for our four ROIs

(a) The timecourses of all four seed ROIs are better explained by a regularized voxel-level connectivity map in PPA, rather than a single connectivity weight for all of left and right PPA. Activity in LOC, TOS, and RSC is most closely related to PPA activity, while only a smaller amount of the cIPL timecourse is related to PPA activity. (b) To obtain a simple characterization of the learned maps, we compute the correlation between the connectivity weights and the anterior-posterior axis. This measure shows consistent differences between the four regions' connectivity maps. LOC and TOS are preferentially connected to posterior PPA (since their corresponding PPA weightmaps increase along the anterior to posterior axis) while RSC and cIPL are preferentially connected to anterior PPA. Error bars represent s.e.m. across subjects, $* p<0.05, * * p<0.01$. 


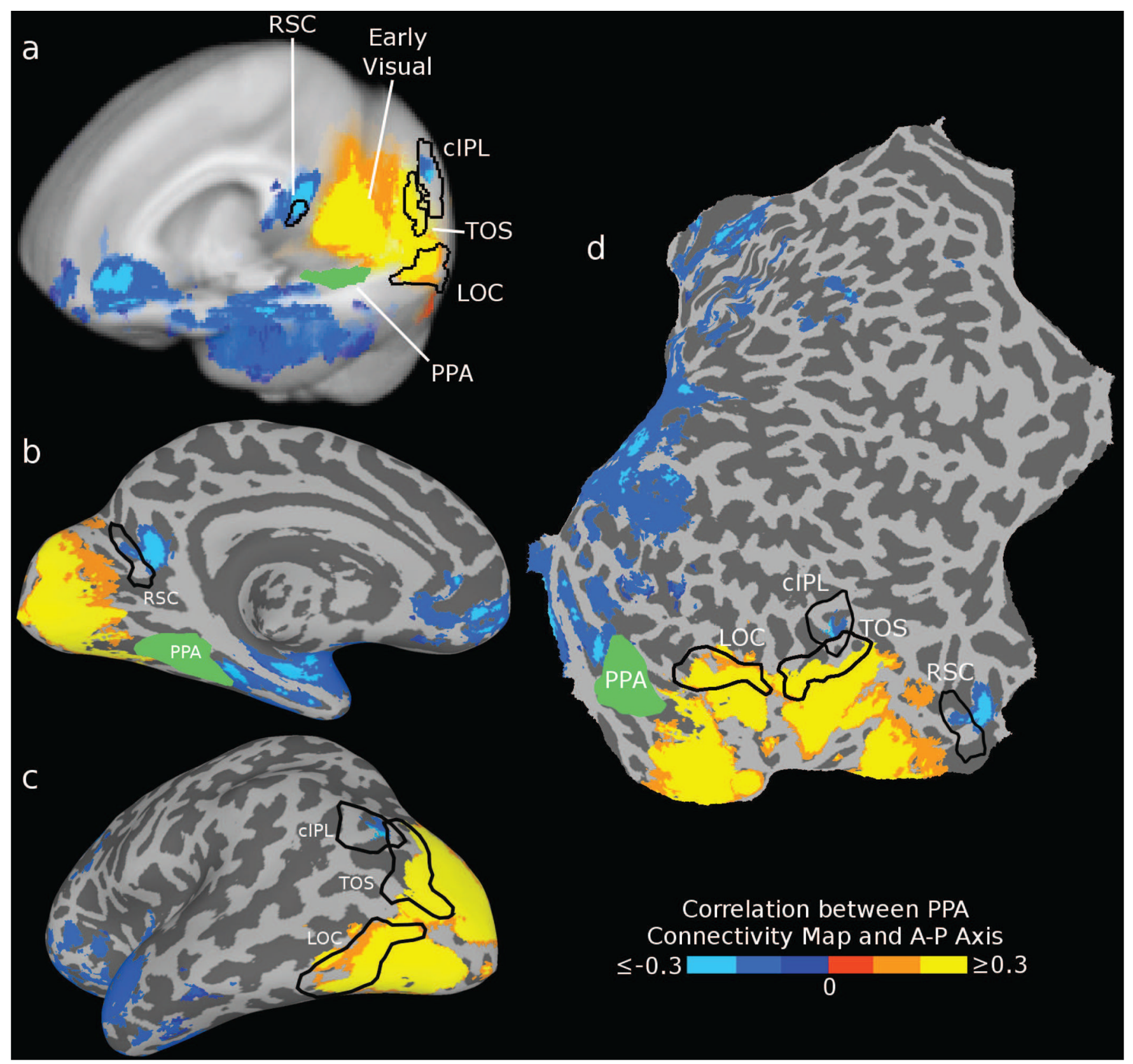

Figure 3. Searchlight connectivity results

(a) Rendering of the group connectivity bias map on the left hemisphere of the Talairach 452 brain. Colored voxels are those that showed highly significant (FDR $<0.01$, cluster size $>$ $300 \mathrm{~mm}^{3}$ ) bias in anterior-posterior connectivity to PPA, computed as the correlation between the learned PPA connectivity map and the anterior-posterior axis. Bilateral areas RSC and cIPL, as well as ventral PFC and lateral anterior temporal regions, exhibited connectivity with anterior PPA (blue voxels), while occipital visual areas (including LOC and TOS) exhibited connectivity with posterior PPA (orange-yellow voxels). The borders of the group ROIs are shown for reference (outlining the location where at least 3 subjects' ROIs overlap). (b-d) The same connectivity map on an inflated surface and cortical flatmap. 


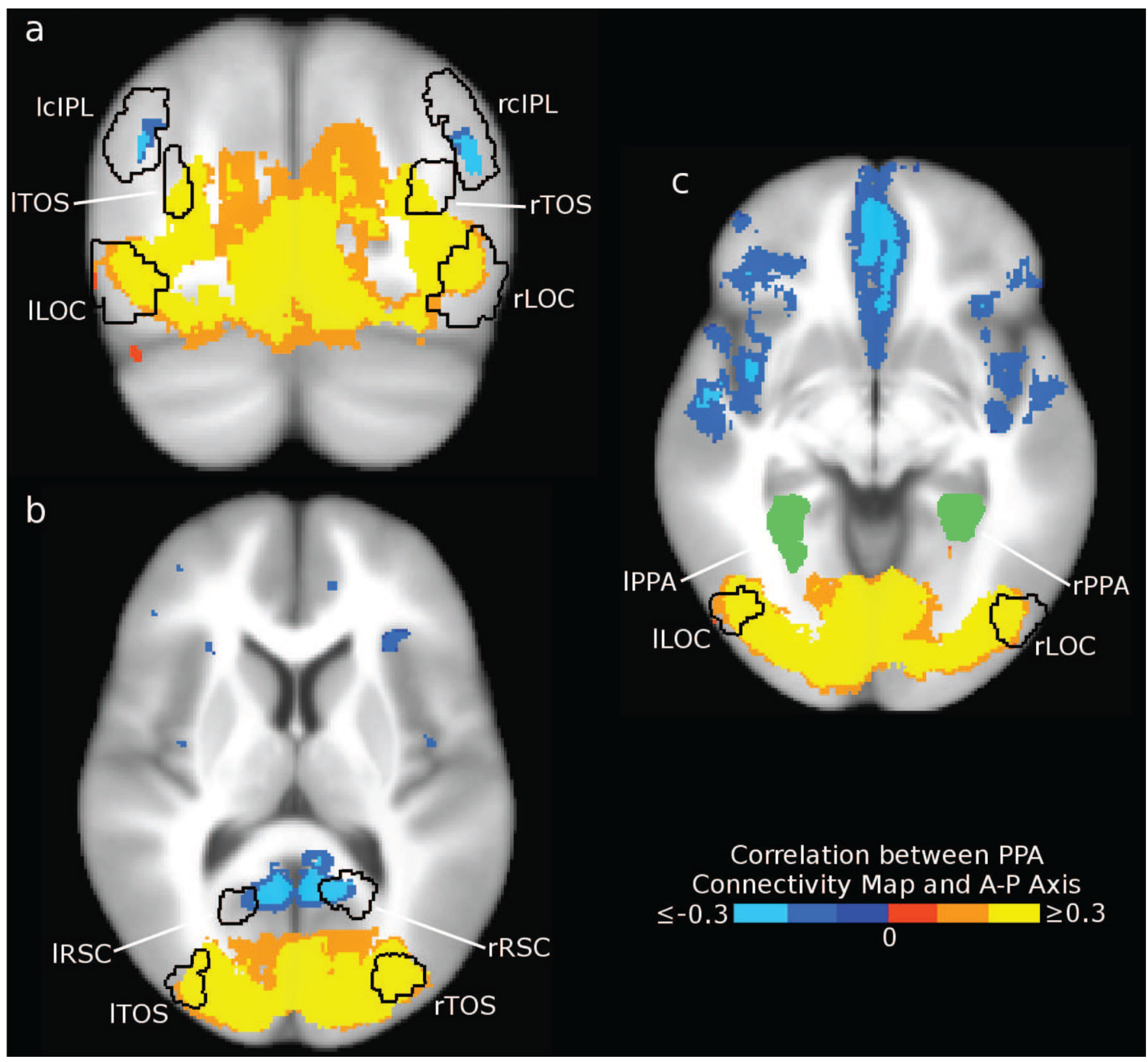

Figure 4. Three slices of the group connectivity bias map

Seed voxels for which the PPA connectivity map has a strong anterior-posterior gradient $\left(\right.$ FDR $<0.01$, cluster size $>300 \mathrm{~mm}^{3}$ ) are shown in blue (preferential connectivity to anterior PPA) and yellow (preferential connectivity to posterior PPA). (a) In this coronal slice ( $y=$ $-73 \mathrm{~mm}$ ), we identify bilateral cIPL regions that show a different connectivity pattern from adjacent area TOS. (b) At $\mathrm{z}=10 \mathrm{~mm}$, we observe anterior PPA connectivity in RSC, as well as posterior PPA connectivity in TOS and early visual visual areas. (c) At $\mathrm{z}=-5 \mathrm{~mm}$, ventral occipital areas including LOC show connectivity to posterior PPA. Additionally, anterior PPA connectivity can be seen in the frontal and anterior temporal lobes. 

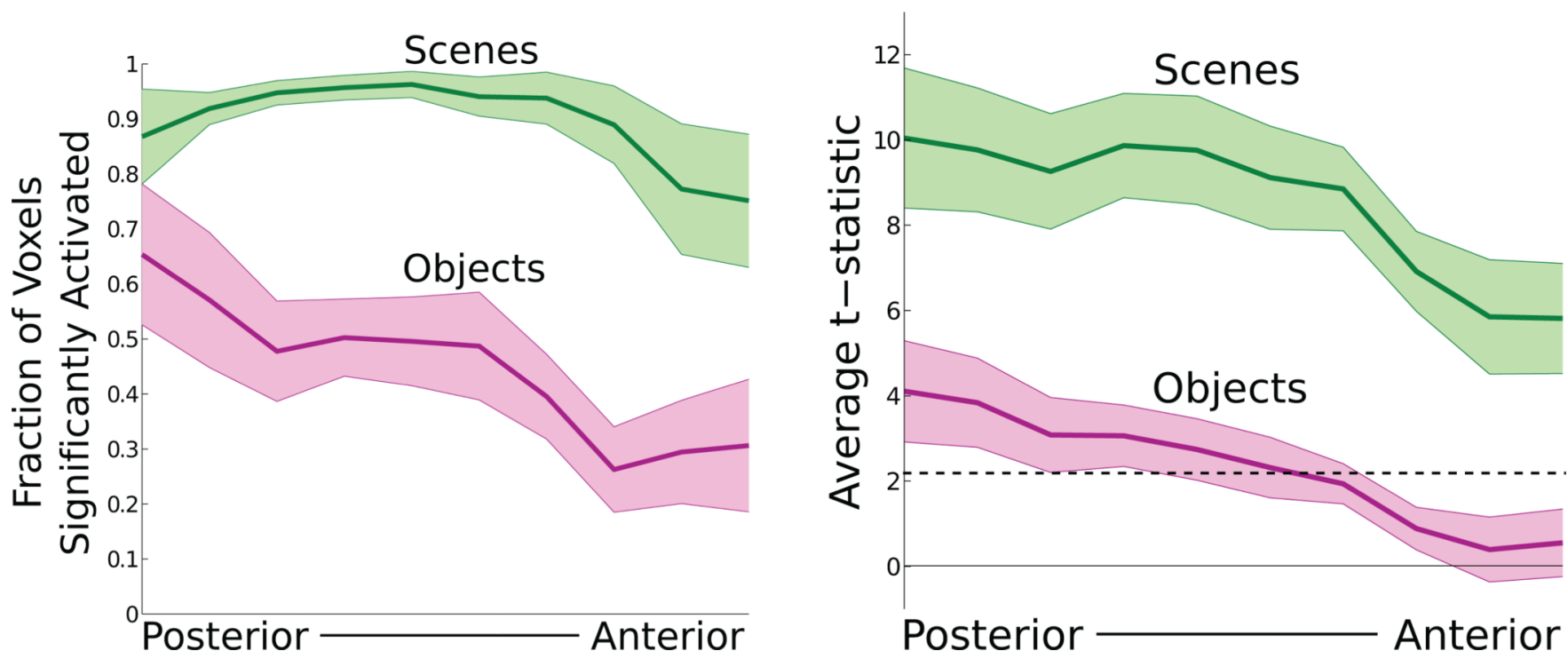

Figure 5. Functional gradients across PPA

The proportion of voxels responsive to scene and object stimuli, and the average t-statistic for the response to scene and object stimuli, were calculated in 10 bins along the anteriorposterior axis in each subject. The dotted line indicates the average t-statistic value corresponding to FDR=0.05 (across all subjects, for both stimulus categories). Scene sensitivity decreased from posterior to anterior PPA, but nearly all voxels across PPA responded significantly to scene stimuli. Object sensitivity substantially decreased from posterior to anterior PPA, with the majority of anterior PPA voxels failing to respond significantly to object stimuli. Error bars represent s.e.m. across subjects. 


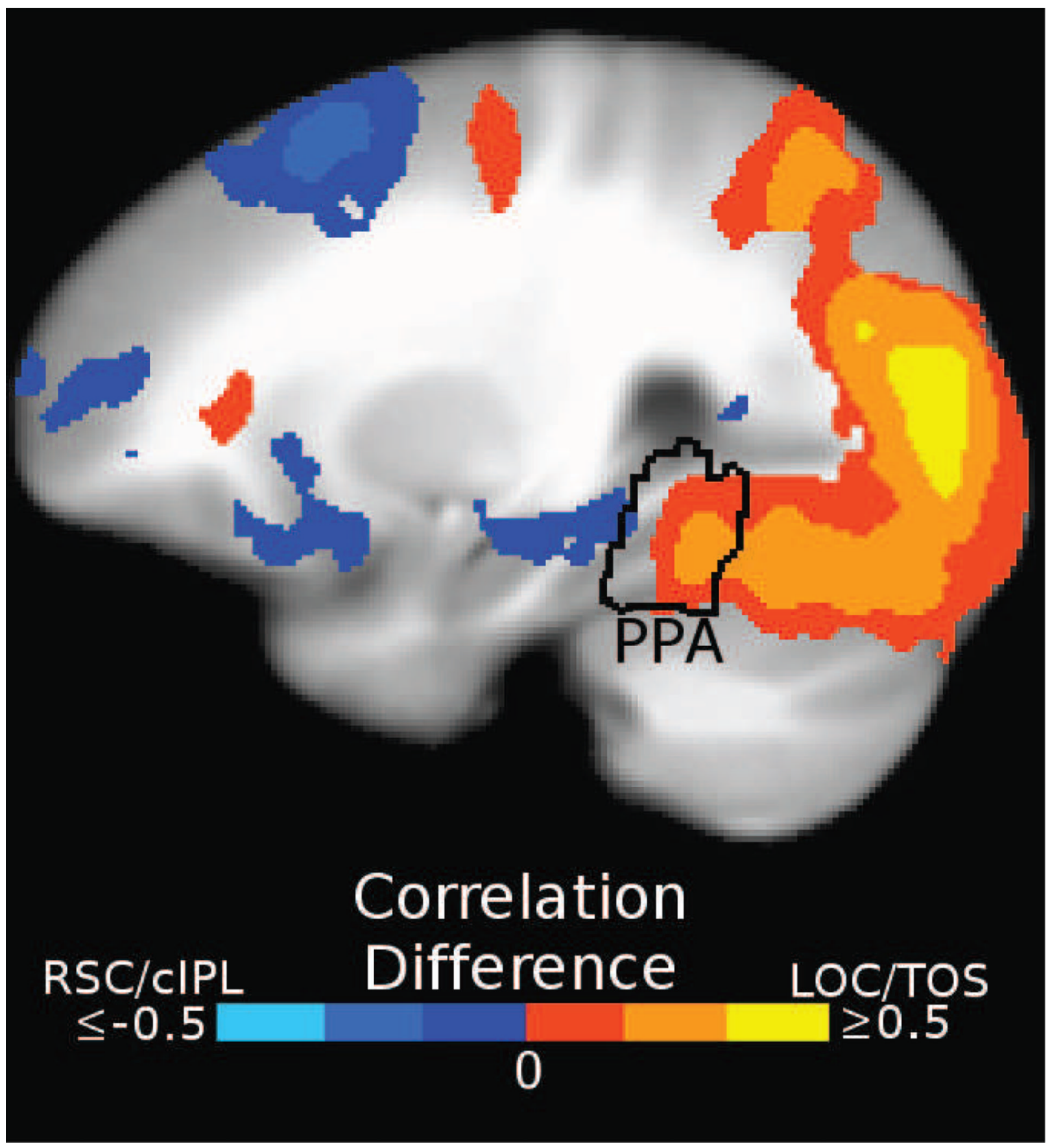

Figure 6. Regions throughout cortex showing connectivity differences similar to anterior and posterior PPA

In this sagittal slice ( $x=-26)$, colored voxels are those showing significantly (FDR $<0.05$, cluster size $>1000 \mathrm{~mm}^{3}$ ) different connectivity to LOC and TOS versus RSC and cIPL. The connectivity pattern in anterior PPA extends anteriorly along the parahippocampal gyrus and into the hippocampus. The connectivity patterns over the entire surface are shown in Supplementary Fig. 6. 